\title{
Nail Wound and Cellulitis Following Typhoon Hagibis in Fukushima, Japan
}

\author{
Akihiko Ozaki, MD, PhD; Yoshiaki Kanemoto, MD; Tomohiro Morita, MD, PhD; \\ Yoshitaka Nishikawa, MD, PhD; Toyoaki Sawano, MD; Sho Fujioka, MD; Yuki Shimada, MD; \\ Asaka Higuchi, RN, PHN, MSN; Masaharu Tsubokura, MD, PhD; Norio Kanzaki, MD
}

Key Words: floods, foot injuries, natural disasters, tetanus

E xtreme weather events, especially flooding, have been on the increase worldwide. ${ }^{1}$ In October 2019, Typhoon Hagibis struck Japan. Excessive rainfall resulted in levees being breached in 128 sections of 71 rivers throughout the country, with approximately 100 people losing their lives, mostly through drowning.

A 46-year-old male presented to our hospital in Iwaki City, Fukushima, with swelling and redness in his right foot (Figure 1a) 3 days after Typhoon Hagibis struck Fukushima. His home was not located in a flood-prone area, but the typhoon caused a lot of water, vegetation, and rubble to accumulate around his house. On examination during the initial presentation, he had a wound, which he reported was a result of a metal nail on his right foot (see Figure 1b). The injury had been caused by contact with a strut sticking out from an old fence block (see Figure 1c). He was wearing running shoes when the injury occurred (see Figure 1d). He attended an emergency room at another hospital immediately after being injured. He was unable to recall whether he had previously received a tetanus vaccination, and the attending physician washed the wound with water, prescribed cefaclor, but did not administer a tetanus vaccination. During 2 days of antibiotic treatment, the patient's injured foot gradually became swollen and red, which led him to visit our hospital.

He was admitted for cellulitis in his injured foot, and intravenous antibiotics (cefazolin $1 \mathrm{~g}$ per 8 hours) and a tetanus vaccination and human anti-tetanus immunoglobulin were administered the same day. The foot X-ray did not show apparent signs of osteomyelitis. Because the patient's infection was limited to the wound and its surrounding area when he initially consulted our department, and the patient had already begun a course of antibiotics, we judged that his condition did not necessitate microbial culture examination of the wound. His injury responded to treatment, and he was discharged on the sixth day after admission.

This case highlights the importance of using proper clothing and equipment when clearing up after natural disasters. ${ }^{1,2}$ Wounds from exposed nails and other sharp objects or surfaces can be difficult to cleanse and can easily become infected. This case emphasizes that the use of appropriate protective footwear and clothing can prevent accidents, potentially injury and subsequent infections.

It is notable that the patient failed to receive a tetanus vaccination during his visit for emergency medical help. Tetanus is an acute, vaccine-preventable infectious disease, arising through infection with spores of Clostridium tetani, a common soil-dwelling bacterium, with the infection frequently occurring in minor and/or clean wounds. ${ }^{3}$ The incidence of tetanus is low in resource-rich settings. ${ }^{4}$ Nevertheless, more than 2000 cases were observed in the United States in $2016,{ }^{5}$ and approximately 100 cases of tetanus occur annually in Japan. ${ }^{3}$ Consequently, its risk should not be underestimated. Although the survival rate from the disease is now more than $90 \%$, tetanus cases are known to increase after natural disasters. It has been suggested that tetanus vaccination should routinely be given to patients with wounds in cases where they cannot recall whether their tetanus vaccinations are complete and up to date. ${ }^{6}$

In Japan, a 1968 law requires universal vaccination against diphtheria, pertussis, and tetanus. The primary infant course of 3 injections provides good protection for a number of years, whereas the fourth and fifth booster doses give lifelong protection. Individuals should be aware of their vaccination status, particularly when they engage in activity that puts them at risk of infection. ${ }^{2}$ However, because it is not always possible to do so, particularly in the immediate aftermath of disasters, health workers should be aware of the importance of administering a tetanus vaccination as soon as possible.

We believe that this case has additional important implications, given the local context of Fukushima Prefecture. During Typhoon Hagibis, Fukushima Prefecture was particularly badly hit, the prefecture having previously experienced the 2011 triple disaster 
(a) Patient's Right Foot with Swelling and Redness. (b) Nail Wound in the Patient's Right Sole (arrow). (c) Strut Projecting from the Old Block Fence in the Backyard of the Patient's Residence (arrow). (d) Shoes Worn by the Patient at the Time of the Injury.

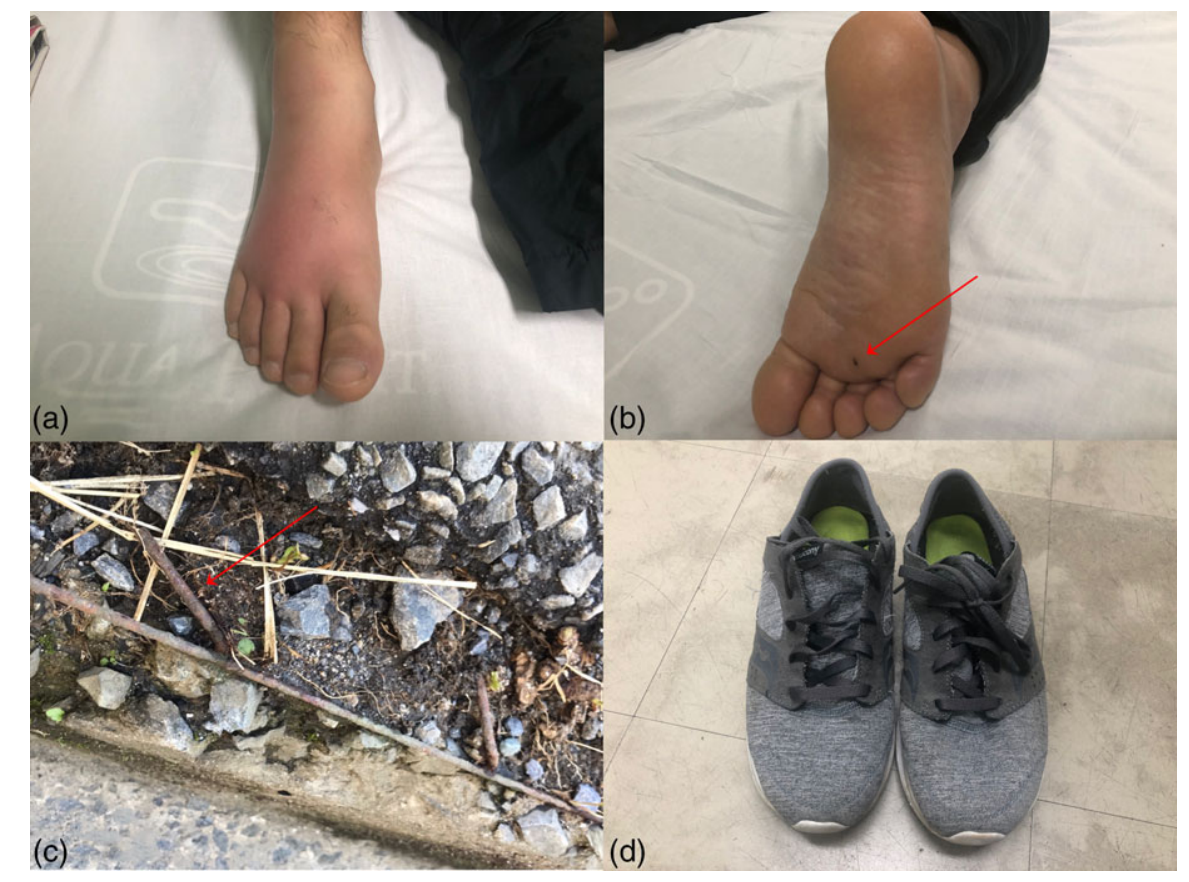

(major earthquake, tsunami, and nuclear accident). ${ }^{7}$ Given that Japan did not provide universal tetanus vaccination before $1968,{ }^{8}$ those born earlier - that is, age 52 years and above at the end of 2019 - have a relatively high risk of tetanus infection. According to a report published in February, the number of Fukushima residents age 55 years and above was 831914 , accounting for $45.3 \%$ of the total population. Given these facts, a limited proportion of Fukushima's elderly population is assumed to have a protective level of anti-tetanus antibody, ${ }^{9}$ and thus the importance of routine tetanus vaccination or boosters cannot be overemphasized. However, given that there are other critical health issues in post-disaster Fukushima, ${ }^{10,11}$ it is important for all relevant stakeholders to determine an appropriate, cost-effective health intervention policy suitable for meeting the needs prevailing at present.

\section{About the Authors}

Department of Breast Surgery, Jyoban Hospital of Tokiwa Foundation, Iwaki, Fukushima, Japan (Dr Ozaki); Department of Surgery, Jyoban Hospital of Tokiwa Foundation, Iwaki, Fukushima, Japan (Drs Kanemoto, Kanzaki); Department of Internal Medicine, Soma Central Hospital, Soma, Fukushima, Japan (Drs Morita, Nishikawa, Fujioka, Tsubokura); Department of Public Health, Fukushima Medical University School of Medicine, Fukushima, Japan (Drs Sawano, Tsubokura); Department of Surgery, Sendai City Medical Center, Sendai Open Hospital, Sendai, Miyagi, Japan (Dr Sawano); Department of Neurosurgery, Minamisoma Municipal General Hospital, Minamisoma, Fukushima, Japan (Dr Shimada) and Medical Governance Research Institute, Minato-ku, Tokyo, Japan (Ms Higuchi).
Correspondence and reprint requests to Akihiko Ozaki, Department of Breast Surgery, Jyoban Hospital of Tokiwa Foundation, 57 Jyoban-KamiyunagaYamachi, Iwaki, 972-8322, Japan (e-mail: aozaki-tky@umin.ac.jp).

\section{Ethics Statement}

The Jyoban Hospital of Tokiwa Foundation Institutional Review Board waived review because this is a case report. Prior informed consent was obtained from the patient concerned for publication of the case study.

\section{Author Contributions}

AO wrote the manuscript. All authors conceptualized and designed the study and revised the paper.

\section{Conflict of Interest Statement}

AO reports receiving a personal fee from MNES Inc. outside the scope of the submitted work.

\section{Acknowledgments}

We express our gratitude to Professor Andy Crump for his constructive opinion on this study and for assistance in preparing the manuscript for publication.

\section{REFERENCES}

1. Alderman K, Turner Lr, Tong S. Floods and human health: a systematic review. Environ Int. 2012;47:37-47.

2. Morita T, Tsubokura M, Tanimoto T, et al. A need for tetanus vaccination before restoration activities in Fukushima, Japan. Disaster Med Public Health Prep. 2014;8:467-468.

3. The Topic of This Month (Tetanus) [In Japanese]. IASR. 2008;30:65-66. 
4. Sexton DJ. Tetanus. In: Bartlett JG, Sullivan M, eds. UpToDate. Waltham, MA: UpToDate. n. d. https://www.uptodate.com/contents/tetanus? search $=$ Tetanus\&_source $=$ search $\_$result $\&$ selectedTitle $=1 \sim 150 \&$ usage $\_$ type $=$ default $\&$ display_rank=1. Accessed March 8, 2020.

5. Liang Jl, Tiwari T, Moro P, et al. Prevention of pertussis, tetanus, and diphtheria with vaccines in the United States: recommendations of the Advisory Committee on Immunization Practices (ACIP). MMWR Recomm Rep. 2018;67:1-44.

6. Hibberd Pl. Tetanus-diphtheria toxoid vaccination in adults. In: Weller PF, Sullivan M, eds. UpToDate. Waltham, MA: UpToDate. n. d. https://www.uptodate.com/contents/tetanus-diphtheria-toxoidvaccination-in-adults?search $=$ Tetanus-diphtheria $\% 20$ toxoid\&source= search_result\&selectedTitle $=2 \sim 148 \&$ usage_type $=$ default\&display_rank $=1$. Accessed March 8, 2020.
7. Morita T, Nomura S, Furutani T, et al. Demographic transition and factors associated with remaining in place after the 2011 Fukushima nuclear disaster and related evacuation orders. PLoS One. 2018;13:E0194134.

8. Nakano T. Japanese vaccinations and practices, with particular attention to polio and pertussis. Travel Med Infect Dis. 2011;9:169-175.

9. McQuillan GM, Kruszon-Moran D, Deforest A, et al. Serologic immunity to diphtheria and tetanus in the United States. Ann Intern Med. 2002;136:660-666

10. Ozaki A, Nomura S, Leppold C, et al. Breast cancer patient delay in Fukushima, Japan following the 2011 triple disaster: a long-term retrospective study. BMC Cancer. 2017;17:423.

11. Nomura S, Blangiardo M, Tsubokura M, et al. Postnuclear disaster evacuation and chronic health in adults in Fukushima, Japan: a long-term retrospective analysis. BMJ Open. 2016;6:E010080. 